\title{
Some of the brain mechanisms of the state of induced relaxation
}

\author{
Popova Tatiana Vladimirovna*, Koryukalov Yury Igorevich, Kourova Olga Germanovna
}

Dep. of Management and Business of National Research University «South Ural State University», Chelyabinsk, Russia

\section{Email address:}

tati.popova2010@yandex.ru (T.V. Popova)

\section{To cite this article:}

Popova Tatiana Vladimirovna, Koryukalov Yury Igorevich, Kourova Olga Germanovna. Some of the Brain Mechanisms of the State of Induced Relaxation. Advances in Bioscience and Bioengineering. Vol. 2, No. 2, 2014, pp. 8-13. doi: 10.11648/j.abb.20140202.11

\begin{abstract}
We have examined 23 teenage males that attended relaxation classes for two years. The subjects practiced two relaxation techniques by doing two different exercises. Each of them had their electroencephalography (EEG) recorded during each exercise. We have detected changes in the electrical activity of their brains, which depended on either of the two exercises done to produce relaxation induction, namely, 1- visualizing images of nature, or 2 - concentrating on the projection of the subject's own body. The alpha activity reached its peak value of 8 to $9 \mathrm{~Hz}$ when doing exercise 1 , while brain electricity synchronization in central and occipital areas of the cortex was predominant when doing exercise 2, which indicated that there existed close interaction between visual and somatosensory cortical projections.
\end{abstract}

Keywords: Relaxation Techniques, Electroencephalography, University Students

\section{Introduction}

A human body is constantly subject to action of assorted information coming from both the outer world and the internal organs. All the sense organs receive signals that include colors, smells, sounds, electric impulses from the heart and the muscles, etc. A human body receives and processes those signals - on the subconscious level - that is, below the level of conscious perception, and the brain puts up barriers to keep most of the signals out. Body responses are generally manifested on an emotional level only [Shulginov A.A., 2002], with functional strain (that is, stress) building up gradually. Relaxation exercises are practiced to prevent stresses.

The studies of psychophysical self-regulation techniques assisted by electrophysiological methods show their effective impact on various psychophysiological processes through changes in functional state of the central nervous system [Aftanas, L.I. 2003; Bundzen P.V. 2000; Gorev A.S., Semenova O. 2003; Lyubimov, N.N. 1999; Popova., T.V. 2004].

However, this is when psycho-emotional state diagnostic testing may be problematic. Psychophysiology implements the recording of nerve impulse and skin electricity, as well as EEG, EMG, and EKG [Davidson, R.J.; Fox N.A. 1982].

On the other hand, gas discharge visualization (GDV) technique is used to provide information about psycho emotional state of an individual [Korotkov, K.G., Korotkina S.A., 1998]. Due to the sensitivity to slightest changes in the body state, the technique is ideal for the research into stress-related states, emotional states, and altered states of consciousness (ASC).

K. G. Korotkov and coworkers have carried out body state diagnostic tests by using typical images of corona discharges on human fingertips. The corona discharged emerged when a finger was put between two electrodes, each carrying high-frequency low-voltage current. GDV technique is currently applied, alongside with EEG, to diagnose a wide range of human body functional states.

The technique in question is also of interest when applied to teaching self-regulation and relaxation techniques, as it has provided proven state of relaxation.

The results of psychoregulation patterns in the last thirty years have served as convincing evidence that the regular practice of psychophysical exercise (PPE) leads to persistent changes in a person's psychophysiological status [Gaylord C., et all, 1989; Popova T.V., Koryukalov Y.I. 2003].

Visualization (concentration of one's attention on illusory visual images) is a major component of PPE. It allows to 
achieve the altered state of consciousness (ASC) faster and to a greater degree. The research of visualization mechanisms is essential to understand the formation of ASC.

The goal of this research is to study the psychophysiological patterns of ASC-related concentration and visualization based on bioelectric activity data of the brain.

\section{Methodology}

The test subjects were selected from university students between 17 and 22 years of age (11 subjects) practicing psychophysical self-regulation for at least 1 year (group 1). The reference group included subjects of the same age and gender (12 subjects) who never practiced PPE before.

The subjects were asked to carefully examine a landscape photo until they could form its complete image in their mind. Then they were asked to visualize the landscape and transfer their consciousness (I) into the visualized image.

GDV technique is being utilized for an increasingly wider range of scientific and practical applications, including medicine and sport, as well as water studies, botany, and mineralogy. An object under studies is placed in a high-tension electromagnetic field to simulate electronic and photonic emission from the surface of the object. Charged particles breed in the gas discharge; an image of corona discharge is then transferred to the PC to be analyzed [Korotkov K G. , 2007; Korotkov K. et. al. 2010]. Kirlian photographs of all the ten human fingers provide detailed information about the individual and their somatopsychic and physicological state [ Yakovleva E.G , 2012; Hossu M, Rupert R. 2006]. The fields of application of GDV techniques include medicine, sport, water studies, and material studies.

The computer EEG included the spectral and correlation analysis. We used the multichannel EEG registration from bowl-shaped electrodes connected to ear electrodes and placed strictly according to the 10-20 system. We took several functional tests: baseline recording (BR), eyes closed (EC), eyes open (EO), examination and visualization of the landscape during the relaxation, baseline recording after leaving the state of relaxation. The EEG sampling rate was $250 \mathrm{~Hz}$.

\section{Results}

During the examination of the landscape photo the subjects showed no significant changes in alpha-activity compared to the baseline. At the same time the subjects with pronounced baseline alpha-activity (relaxation group) showed inhibition of alpha-activity primarily in occipital and central areas during the examination of the landscape. The received data reflect our hypothesis [Mulholland T., 1969] that the inhibition of alpha-activity under the influence of attention is mediated by the activity of the oculomotor system. An adjacent theory by A.F. Iznak claiming that the higher alpha-activity level reflects the lower level of oculomotor functions activation and of handeye integration [Iznak A.F. 1989; Iznak A.F., Chaianov N.V. 1989].

The analysis of data received from histograms and sweeps during the eyes closed (EC) test revealed the flow of alpha-wave activity primarily in occipital and frontal areas focused on occipital areas with amplitude and volume of alpha activity predominating in the right hemisphere. (fig.1)

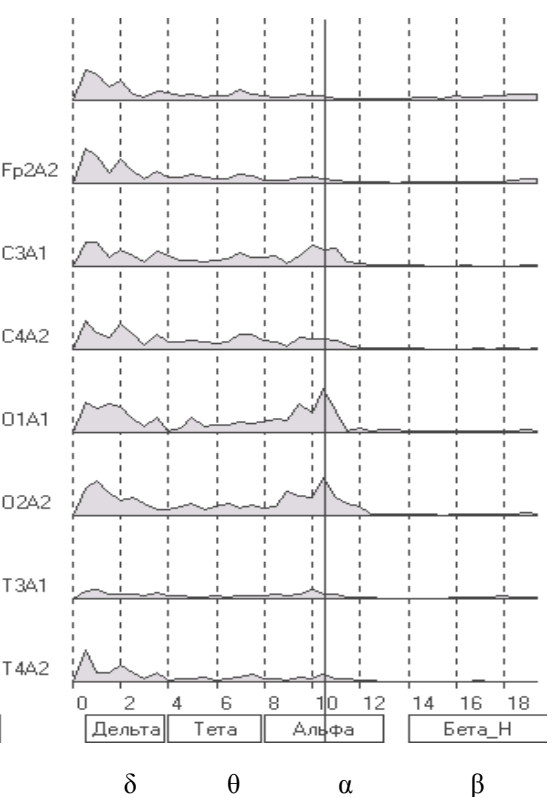

Fig. 1. Dynamics of alpha-wave activity during the eyes closed test

The data reflects the idea of major role of alpha-activity in processing of sensory information in the cerebral cortex
[Basar E. 1980; Basar E, et.all 2003]; specifically, the scanning in the optic cortex that was theoretically forecast 
[Pitts W., McCulloch W.S. 1947] and then experimentally proven [Shevelev I.A., et.all 2003; Shevelev I.A., Tsicalov E.N. 1997].

The process of visualization was accompanied by significant changes in spectral volume (SV) of alpha3activity $(10,5-14 \mathrm{~Hz})$ and a mild increase of SV of alpha2activity $(8,5-10 \mathrm{~Hz})$ dominating in the frontal and occipital areas of the right hemisphere. The most experienced subjects showed the shift of peaking alpha-activity towards the slow-wave activity originating from the frontal area. (fig.2). The fall of the frequency was found with other leads as well. These facts speak of the leading role of frontal areas in regulating the cortical arousal in the mechanism of forming the state of relaxation with visualization of various spatial images.

The subjects were able to reproduce the full spatial image of a visualized object while concentrating their attention on this image and noting their relaxed state of detachment. Supported by the subjects' feelings, the data of sweeps and maps is evidence to the synchronization and generalization of alpha-activity in all EEG leads reflecting the unified activity of neural networks of respective cortex areas while producing a spatial image. We may assume that the visualization helps to achieve a quicker and deeper altered state of consciousness.

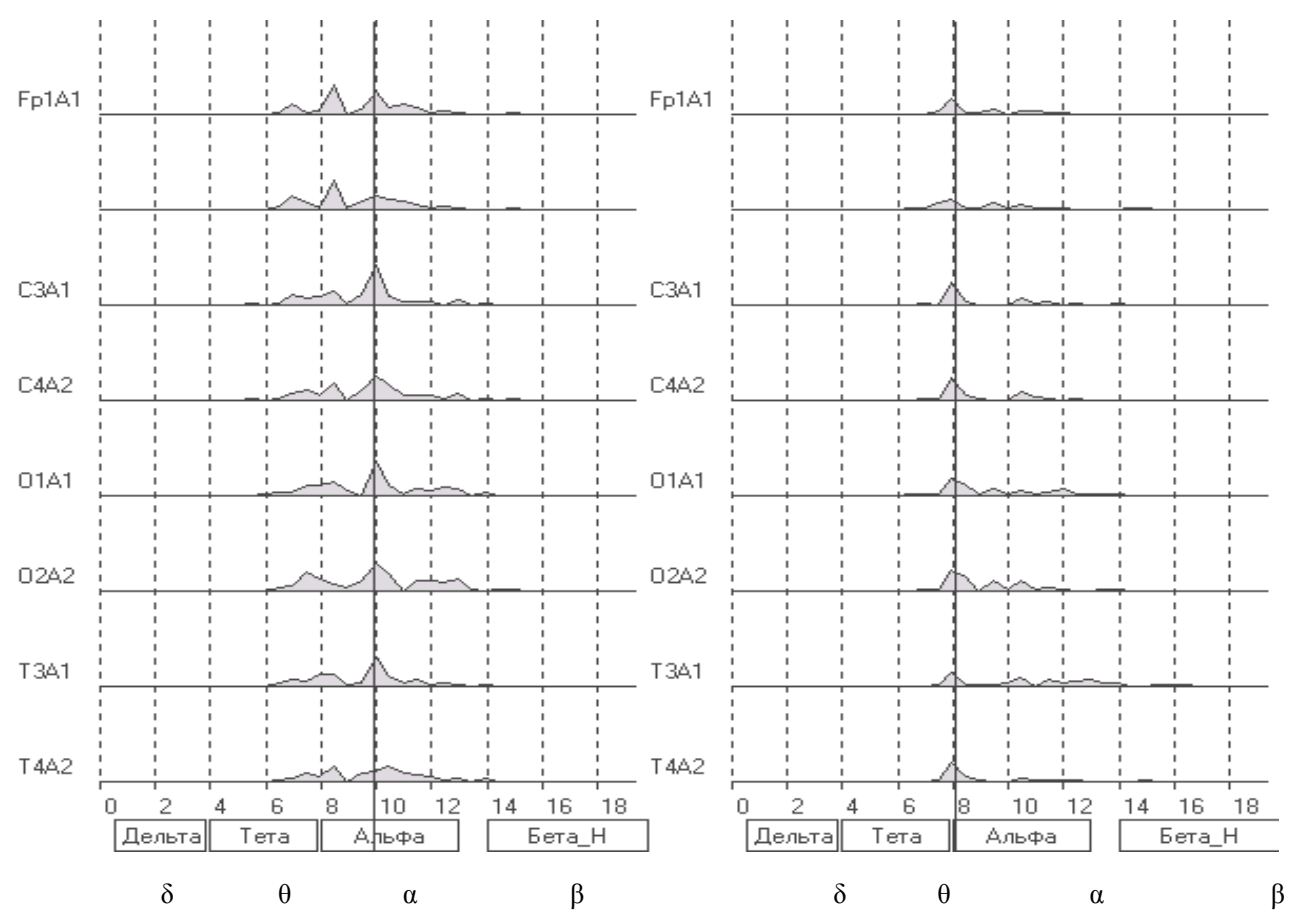

Fig. 2. Shift of peaking alpha activity towards the slow-wave activity during the relaxed visualization (118p-194p epoch 29-30)

Interesting results were obtained by us earlier [Popova T.V. et al, 2002] about the impact of PPE s on the energy state of a person (by the traditional method of visualizing gas (GRV)). Radiation fingers tested before and after the PPE differ in nature, size and form of light. Thus, after the PPE to the relaxation of visualization in 8 of 11 subjects experienced a significant increase in the area of luminescence and the harmonization of forms by filling empty sectors radiation. The remaining subjects with less than three months, PPE, also show changes in light, but in a less pronounced form. Similar phenomena in the changing light fingers subjects occur in the performance of other PFCs. For example, with PPE in the mood for relaxation with almost all subjects, there was «improvement» glow (closer to type I fig.3 ). Development of fatigue caused by inhibition in the central nervous system, combined with a reduction in the emission area of GDV. Our data showed that while there was an increase of fractality on GDV.

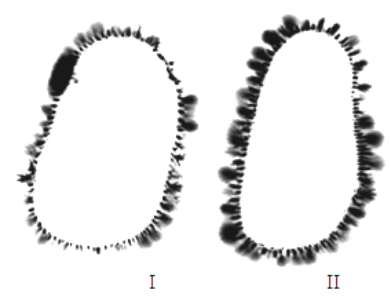

Fig. 3. 3 types radiations of fingers

The analysis of EEG instantaneous amplitude says that hemispheres reported synchronized activity during the initial stage $(10-20 \mathrm{sec})$ of visualizing the natural landscape. The next stage when a subject needed to integrate themselves into the visualized place showed a slight prevalence of the right hemisphere's activity; this was followed by a significant increase in the activity of occipital areas of predominantly right hemisphere. Compared to the previous stage, the increase in activity was found also in the central areas - a projection of somesthetic 
area. The synchronized activity was found within the respective period at all EEG leads with maximum amplitude in central and occipital areas of both hemispheres and in the frontal neocortex of the right hemisphere (fig.4).
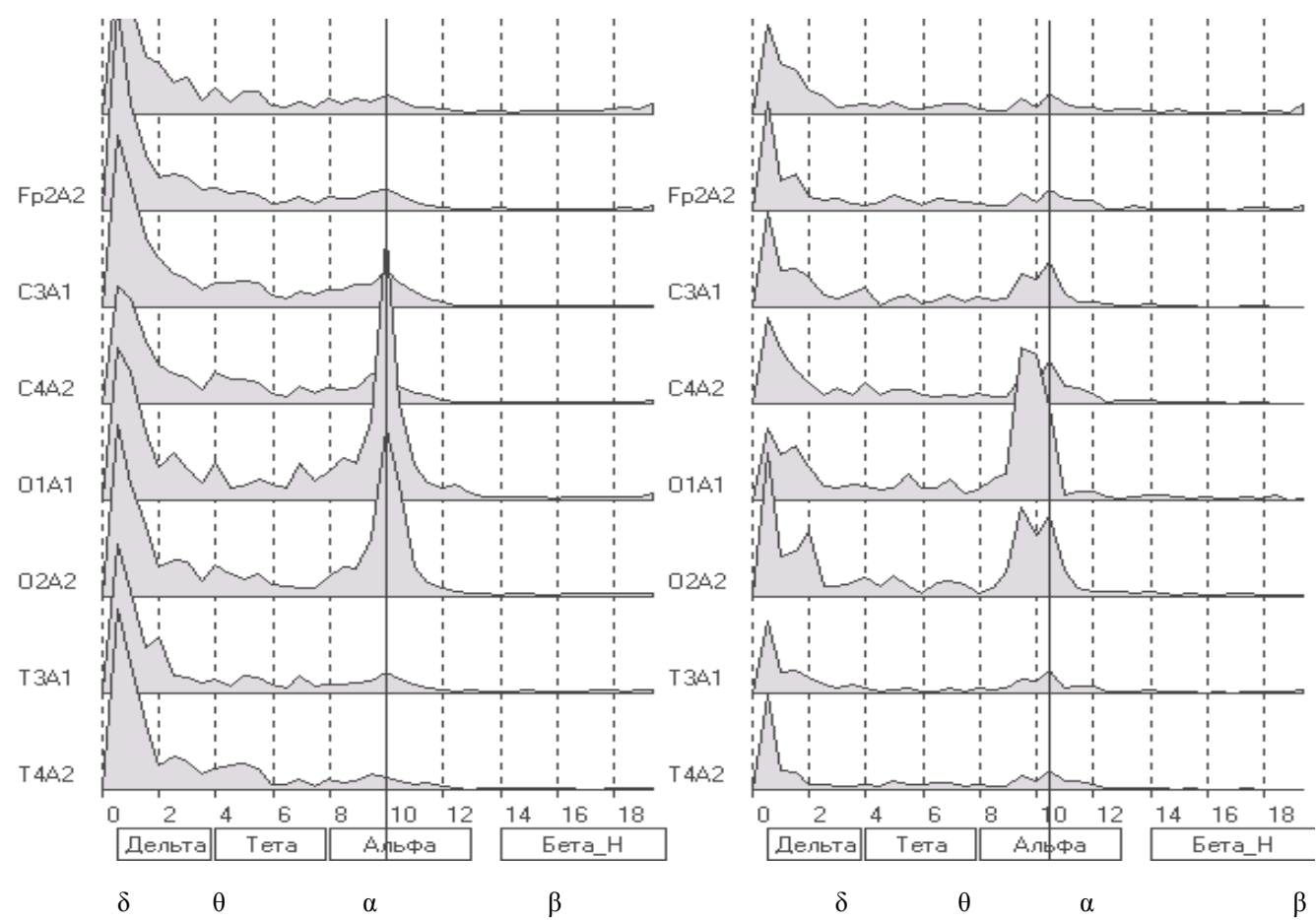

Fig 4. Dynamics of alpha-wave activity during the second stage of visualization. Subject Sh-va 21years old

The facts are speaking of a single neural network between the optical and somesthetic areas at the moment of combined optical perception of a spatial image and the concentrated attention on somatosensation in the imaginary space.

After the end of visualization the alpha-activity was inhibited though its internal and hemispheric synchronization remained (fig 5), which was especially manifested for subjects with expressed alpha-activity.
Evidently we may speak of preservation of functional connections of neural network that form in the altered state of consciousness and incorporate the respective parts of the cortex in psychic activity not just for the duration of relaxation but also after it has passed. Perhaps this mechanism explains the development of creative capabilities in people that regularly practice relaxation and meditation techniques.

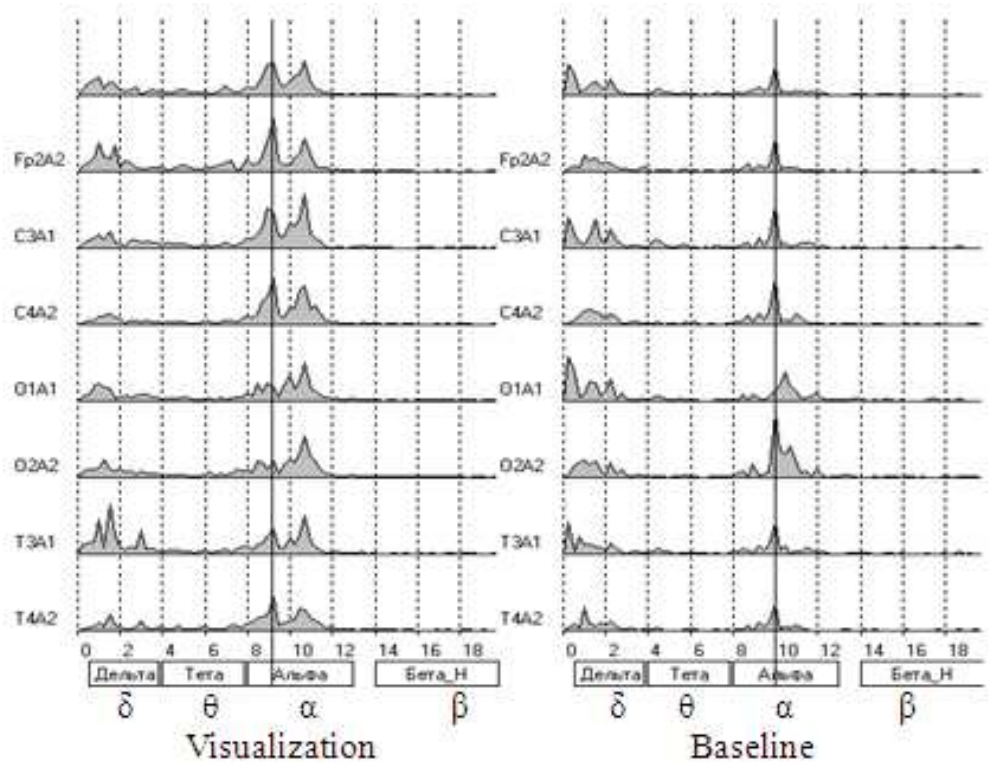

Fig 5. Changes in alpha-activity volume after the end of visualization. 


\section{Conclusions}

1. The subjects of the relaxation group showed a second peak zone of low-frequency $(9 \mathrm{~Hz})$ alpha-activity during the visualization PPE. After the end of visualization the alpha-activity was inhibited though its internal and hemispheric synchronization remained for subjects with expressed alpha-activity.

2. The synchronization of bioelectric activity between the central and occipital regions during the body projection PPE leads to formation of a single functional neural network of visual and somesthetic zones.

\section{References}

[1] Aftanas, L.I. [2003] The dynamics of cortical activity in altered states of consciousness: a study meditation with the help of high-resolution EEG [Text] / LI Aftanas, SA Golosheykin / / Human Physiology. - V. 29, № 2. - P. 18-27.

[2] Bundzen P.V. [2000] Current trends in the development of technologies mental training of athletes / / Annual Scientific Bulletin State Academy FC named. PF Lesgaft. St. Petersburg., 2000, P. 40 - 44.

[3] Gorev A.S., Semenova O. [2003] The influence of individual characteristics on the effectiveness of CNS formation of relaxation skills in the use of biofeedback in children 9-10 years old / / Human Physiology, 2003, v.29, № 4. P.54-61.

[4] Davidson, R.J., Fox N.A. Asymmetrical brain activity discriminates between positive versus negative affective stimuli in human infants, Science, Vol. 218, 1982, pp. 12351237

[5] Iznak A.F. [1989] Modulation of sensorimotor activity against human alpha-rhythm EEG / / Problems of scientific. Tests were performed. in the region. psychol. Health / Ministry of Health of the USSR Academy of Medical Sciences of the USSR. - 1989. - P. 3-24.

[6] Iznak A.F., Chaianov N.V. [1989] Subjective correlates of outbreaks of the alpha rhythm in the human EEG during visual-motor operator activity / / Problems of scientific. Tests were performed. in the region. psychol. Health / Ministry of Health of the USSR Academy of Medical Sciences of the USSR. - 1989. - Pp. 24-30.

[7] Lyubimov, N.N. [1999] Changes in EEG and evoked potentials in the special form of psychological training / NN Lyubimov / / Human Physiology. - 1999. - V. 25, № 2. - P. $62-66$

[8] Popova T.V., Koryukalov Y.I. [2003] Bioelectrical activity of the brain in a state of relaxation in students 17-23 years old / Bulletin of the South Ural State University. Series "Education, health, physical culture", 2003, Issue 2, № 5 (21), $130 \mathrm{p}$.

[9] Popova T.V. [2004] The mechanism of relaxation induced states / T.V. Popova, YI Koryukalov, OG Kourova / / Bulletin of the South Ural State University. Ser. Education, health, physical education. - Issue. Number 6 (b). -
Chelyabinsk, 2004. - P. 64-67.

[10] Basar E. [1980] EEG dynamics: relations between EEG and evoked potentials. // Elservier. 1980, North-Holland,New York.P.37-49.

[11] Basar E, Schurmann M, Basar-Eroglu C, Karakas S. [2003] Alpha oscil-lations in brain functioning: an integrative theory. //Int.J.Psychophysiol. 1997. V.26. N1-3. P.5-29.

[12] Gaylord C., Orme-Johnson D., Travis F. [1989] The effects of the transcendental mediation technique and progressive muscle relaxation on EEC coherence, stress reactivity, and mental health in black adults // Int. J. Neurosci. 1989. V. 46. JVs 1-2. P. 77.

[13] Korotkov, K.G., Korotkina S.A. Application of gas-discharge visualization method for hardware testing of the energyinformation effects level, Kirlian effect to bioelektrografii, Collected articles. Olga, St. Petersburg, 1998.

[14] Korotkov K., Korotkin D. Concentration dependence of gas discharge around drops of inorganic electrolytes // J of Applied Physics, Vol. 89, № 9, 2001, pp. 4732-4737.

[15] Korotkov K. et. al. The research of the time dynamics of the gas discharge around drops of liquids // J of Applied Physics, Vol. 95, № 7, 2004, pp. 3334-3338.

[16] Korotkov K. Analysis principles in GDV. - St. Petersburg, Moscow, "Renome", 2007, 286 p. Korotkov K.G., Matravers P., Orlov D.V., Williams B.O. Application of Electrophoton Capture (EPC) Analysis Based on Gas Discharge Visualization (GDV) Technique in Medicine: A Systematic Review // J Altern Complement Med., № 16, 2010, pp. 13-25.

[17] Mulholland T. [1969] The concept of attention and the electroencephalographic alpha rhythm // Attention in Psychophysiology / C.R.Evans, T.B.Mulholland (Eds.). - L.: Butterworths. - 1969. - P. 100-127.

[18] Panjwani U., Gupta H.L., Singh S.H. el al. [1995] Effect of Sahaja yoga practice on stress management in patients of epilepsy // Indian J. Physiol. Pharmacol. 1995. V. 39 (2). P. 111.

[19] Pitts W., McCulloch W.S. [1947] How we know universals. The perception of auditory and visual forms //Bull. Math. Biophys. 1947. V.9. P.127-147.

[20] Hossu M, Rupert R. Quantum Events of Biophoton Emission Associated with Complementary and Alternative Medicine Therapies. J AlternComplementMed. 2006, 12(2): 119-124.

[21] Shevelev I.A., Kamenkovich V.M., Kostelianetz N.B., Sharaev G.A. [2003] Recognition of direction of unforms and accelerated visual motion and EEG alpha-wave phases. // FEBS Letters, 1996, V.392,P 169-174.

[22] Shevelev I.A., Tsicalov E.N. [1997] Fast thermal waves spreading over the cerebral cortex// Neuroscince. 1997. V.76. N2. P.531-540.

[23] Shulginov AA, Popova T.V., Stadnik O.S., Koryukalov Yu.I., Image analysis of bioelectrograms of human fields, Proceedings of Eurotherm. France, Reims, October 28-30, 2002, pp 8-9. 
[24] Popova T.V., Shulginov A., Stadnik O., Koryucalov Yu. [2002] Image analysis of bioelectrogram of human filds // Visualization, imaging and data analysis in convective heat and mass transfer. Evrotherm 71. Reims, FRANCE, October 28-30, 2002, p. 365-368.
[25] Rgeusskaja GV, Listopadov UI. Medical Technology of Electrophotonics - GDV - in Evaluation of Cognitive Functions. J SciHealingOutcome.2009, 2(5): 16-19.

[26] Yakovleva E.G. GDV method in medicine. - Moscow: Publishing House. "Manager" 2012. 105 pp. 